\title{
Eye movements and identifying words in parafoveal vision
}

\author{
KEITH RAYNER and ROBERT E. MORRISON \\ University of Massachusetts, Amherst, Massachusetts 01003
}

\begin{abstract}
Subjects either named or made lexical decisions about words presented in parafoveal vision. In one condition, subjects were required to maintain fixation, and in another condition, they were allowed to make eye movements. In the no eye movement condition, performance decreased as the stimulus was presented further from fixation. Words could be identified more quickly when eye movements were made than when they were not. The experiments also indicated that holding fixation takes up a certain amount of processing capacity, so that foveally presented targets are identified more quickly when eye movements are allowed than when they are not.
\end{abstract}

Numerous psychophysical experiments have demonstrated that acuity and vision deteriorate as a stimulus is presented at increasing distances from the fovea. While it is not surprising that acuity and vision in general should be relatively poor in the peripheral region, the data also indicate that stimuli presented in parafoveal vision (up to $5 \mathrm{deg}$ from the fixation point) are severely degraded with respect to stimuli presented in the fovea. For example, Koenderink, Bouman, Bueno de Mesquita, and Slappendel (1978) measured contrast detection thresholds for moving spatial sine-wave gratings in foveal and parafoveal vision and found that acuity at $4 \mathrm{deg}$ from fixation was half that in the fovea. Other psychophysical experiments have reported similar steady decreases of sensitivity with increasing eccentricity in the parafovea (Andriessen \& Bouma, 1976; Hilz \& Cavonius, 1974; Hines, 1976; Limb \& Rubinstein, 1977; Millodot, 1966).

In experiments dealing more with perceptual than psychophysical functions, Bouma $(1973,1978)$ and Schiepers (1980) have demonstrated that the ability to identify a word from a $100-\mathrm{msec}$ exposure or to identify a letter (either in isolation or within a word) decreases as the stimulus is presented farther from fixation. Thus, for example, the probability of correctly identifying a letter embedded in the middle of a trigram drops from $100 \%$ in the fovea to approximately $25 \%$ at $5 \mathrm{deg}$ (Bouma, 1978). Likewise, the probability of correctly recognizing a word presented in the fovea is virtually $100 \%$, but it drops to about $60 \%$ for words presented about $4 \mathrm{deg}$ from fixation (Bouma, 1973; Schiepers, 1980). Bouma (1973) argued that poorer acuity alone cannot account for the results obtained, and he suggested that visual interference of a masking type, predominantly acting

This research was supported by Grant HD12727 from the National Institute of Child Health and Human Development We would like to thank Arnold Well and Alexander Pollatsek for their assistance and comments on the paper. Requests for reprints should be addressed to Keith Rayner, Department of Psychology, University of Massachusetts, Amherst, Massachusetts 01003. toward the fovea, is responsible for the poorer performance with more eccentrically presented stimuli. Results of an experiment reported by Mackworth (1965) are consistent with this interpretation and further suggest that lateral masking by adjacent letters is partially responsible for poorer performance in recognizing parafoveally presented letters and words. In addition, response time for correctly identifying a word presented at different eccentricities increases as the word is presented further from fixation. The slope of this function has been variously determined as $90 \mathrm{msec} / \mathrm{deg}$ (Schiepers, 1980) and $140 \mathrm{msec} / \mathrm{deg}$ (Bouma, 1978).

While it is clear that perceptual and psychophysical functions decrease dramatically in the parafovea, the relationship of eye movements to this poorer parafoveal vision is not clear. That is, it is unclear whether there is a tradeoff (in terms of speed of identifying stimuli) between holding fixation and making an eye movement. For stimuli presented close to fixation, identification may be faster if the subject does not make an eye movement, but for stimuli presented farther from fixation, it may be faster (and certainly more accurate) to move the eyes. Thus, since making an eye movement involves a latency period of about 150-200 msec (Rayner, 1978), subjects may be able to identify words presented close to fixation (yet in the parafovea) more rapidly if they do not make a saccade to that location. Because we are interested in the reading process and the importance of parafoveal vision in reading, in the experiments reported here we asked subjects to either name words or make lexical decisions when the words were presented at different eccentricities from the fovea under conditions in which eye movements were or were not made.

\section{METHOD}

\section{Procedure and Apparatus}

Subjects were seated in front of a cathode-ray tube (CRT) controlled by a computer as their eye movements were recorded. Eye movement recording (see Rayner, McConkie, \& Ehrlich, 1978, for a more detailed description of the apparatus) was accomplished via an infrared recording device. A forehead rest 
and a chinrest were used to stabilize the head. After the eye sensors had been appropriately adjusted and calibrated, subjects were informed of the task and given blocks of practice trials. In Experiment 1, subjects were asked to name a word as rapidly and as accurately as possible. In Experiment 2, the task was to make a lexical decision as rapidly as possible. In Experiment 1, 30 high-frequency words were presented in the fovea or 1,3 , or $5 \mathrm{deg}$ left or right of a central fixation point. The words were five letters long and were presented in uppercase, so as to minimize cues from general word shape and dominant letters. We have also used lowercase words in the experiments and obtained similar results in terms of the overall pattern. In Experiment 2, words or nonwords were presented in the fovea or $1,2,3,4$, or $5 \mathrm{deg}$ to the left or right of fixation. In both experiments, stimuli to the right of fixation began a specified distance from fixation and stimuli to the left ended a specified distance from fixation. Thus, for example, an eccentricity of 3 deg meant the stimulus either ended $3 \mathrm{deg}$ from fixation (to the left) or began $3 \mathrm{deg}$ from fixation (to the right). In Experiment 2, 20 highfrequency words were used as word stimuli, and the nonwords were made by replacing the vowels in the words with visually similar consonants. The stimuli were presented in uppercase. In one condition, subjects were allowed to make eye movements to the stimulus and respond to it. In the other condition, subjects were asked to name the word (Experiment 1) or make a lexical decision (Experiment 2) without making an eye movement. In both experiments, the stimuli were presented for $300 \mathrm{msec}$. Thus, in the eye movement condition, the stimulus was present on the CRT for at least $100 \mathrm{msec}$ in most cases after the eye arrived at its target, since the average latency for different subjects was in the range of 140-200 msec.

The subject's eye was $48 \mathrm{~cm}$ from the face of the CRT, and three character spaces equaled $1 \mathrm{deg}$ of visual angle. The CRT had a P-31 phosphor, with the characteristic that removing a character resulted in a drop to $1 \%$ of maximum brightness in $.25 \mathrm{msec}$.

In each experiment, all of the stimuli occurred randomly (but with equal frequency) at each of the visual angles tested. Thus, in Experiment 1 there were 420 trials and in Experiment 2 there were 880 trials. In addition to the two main experiments, some control experiments were carried out, and they will be described later. Three subjects participated in Experiment 1 and seven different subjects participated in Experiment 2. All of the subjects had normal, uncorrected vision.

\section{RESULTS}

The main results of Experiment 1 are shown in Figure 1. All of the subjects showed the same pattern. The data shown in Figure 1 include the latency of the eye as well as the saccade duration in the eye movement condition. Characteristics of these aspects of the data were identical to those reported previously by Rayner (1978) and will not be discussed here. In the eye movement condition, error rates remained relatively consistent across the visual angles, and naming times increased at the rate of approximately $33 \mathrm{msec} / \mathrm{deg}$ of visual angle that the word was presented from fixation. These data perfectly replicate the data reported by Rayner (1978). In the no eye movement condition, accuracy decreased with increasing distance from the fovea, whereas naming time (for correct identifications) increased at the rate of $85 \mathrm{msec} / \mathrm{deg}$. The no eye movement data thus replicate the experiments reported by Bouma (1973, 1978) and Schiepers (1980). The increase in naming time in the present experiment is very similar to the $90-\mathrm{msec} /$ deg slope reported by Schiepers (1980). The reason for

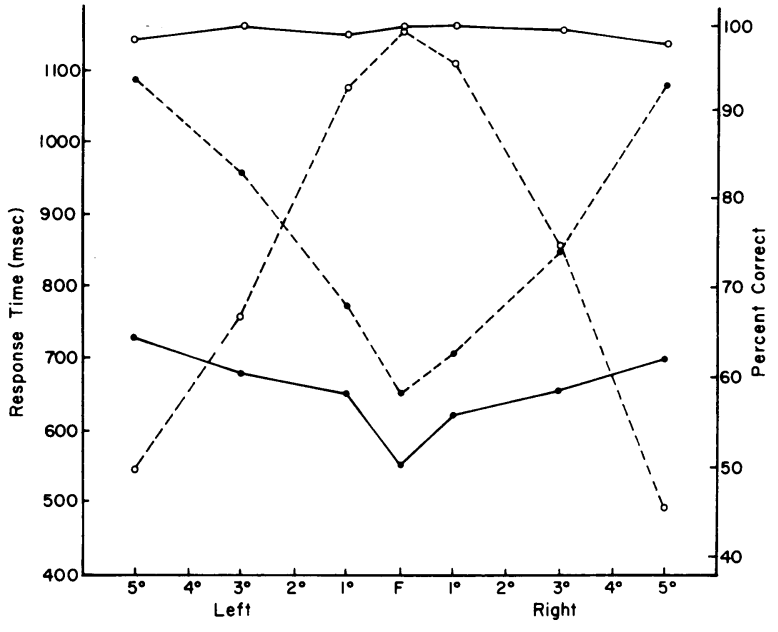

Figure 1. Response time and percent correct in the naming task (Experiment 1). Open circles indicate percent correct, and filled circles indicate response time. Solid lines represent the eye movement condition, and dotted lines represent the no eye movement condition.

the discrepancy between these data and those of Bouma (1978), who found a slope of $140 \mathrm{msec} / \mathrm{deg}$, is not clear.

The effects on naming time and accuracy of two levels of viewing behavior (eye movements and no eye movements) and seven different eccentricities of stimulus presentation (foveal and 1, 3, and 5 deg left and right of fixation) were assessed with separate 2 by 7 analyses of variance. An arcsin transformation was used to normalize the accuracy data.

The critical comparison in the experiment was between the eye movement condition and the no eye movement condition and whether this relationship changed with increasing eccentricity. As Figure 1 shows, naming time and accuracy performance deteriorated rapidly with increasing eccentricity in the no eye movement condition, but in the eye movement condition, naming times increased much less and accuracy did not decline with increasing eccentricity. This pattern results in significant Movement by Eccentricity interactions for naming time $[\mathrm{F}(6,12)=3.95, \mathrm{p}<.05]$ and accuracy $[F(6,12)=30.37, p<.001]$. The main effects of eccentricity $[F(6,12)=14.18, p<.001$, and $F(6,12)=$ $42.67, \mathrm{p}<.001$, for naming time and accuracy, respectively] and movement [reaching significance for the accuracy data only, $F(1,2)=535.23, p<.01]$ also reflect this pattern of data and are indicative of the large effects on performance imposed by holding fixation and by responding without making eye movements. These effects were substantial even at 1 deg from fixation.

Although making an eye movement to a stimulus at $1 \mathrm{deg}$ did seem to cause a large increase in naming time over that found foveally $(60-80 \mathrm{msec}$, far greater than the increase of $15 \mathrm{msec} / \mathrm{deg}$ found beyond $1 \mathrm{deg}$ ), naming time was still better here than in the no eye movement condition. Surprisingly, in the no eye movement condition, naming time was $110 \mathrm{msec}$ longer even 
for foveally presented stimuli. This result was replicated in Experiment 2 and will be discussed further later.

In addition to Experiment 1, two control experiments were conducted with the naming task, using the subjects from Experiment 1. In one experiment, an asterisk was initially displayed 1,3 , or $5 \mathrm{deg}$ from fixation, and during the eye movement the word to be named appeared on the CRT. Thus, at the beginning of the eye movement, an asterisk was displayed, and by the time the saccade had ended, the word to be named was at the target of the saccade. In this experiment, the subject thus had no prior information about the parafoveal word. The results of the experiment showed that the response time curve was flat (mean $=741 \mathrm{msec}$ ) across the different visual angles tested. In the second control experiment, the word was initially presented in parafoveal vision and the display went blank when the subject initiated a saccade to the target. In this condition, the only information that could be used in identifying the parafoveal word was information obtained prior to the eye movement. Subjects were very accurate in identifying words presented $1 \mathrm{deg}$ from fixation (93\%), but at $3 \mathrm{deg}$, accuracy was $61 \%$, and at $5 \mathrm{deg}$, it was $28 \%$. The latency of the eye movement (and hence the amount of time the word was displayed on the CRT) averaged $165 \mathrm{msec}$.

In Experiment 2, we were able to obtain more precise measures concerning accuracy by utilizing a lexical decision task. That is, in Experiment 1, it is difficult to determine the extent to which accuracy performance deviated from chance. Utilization of the lexical decision task made it possible to compare performance at each of the visual angles, since chance performance would be $50 \%$.

The results of Experiment 2 are shown in Figure 2. Again, all of the subjects showed the same pattern. In the eye movement condition, error rates ranged between $4 \%$ and $10 \%$ and were relatively constant across the visual angles tested. Response times were similar to those in Experiment 1, with an increase of $21 \mathrm{msec} / \mathrm{deg}$. In the no eye movement condition, accuracy decreased drastically with increasing visual angle, so that performance on stimuli presented $5 \mathrm{deg}$ from fixation was at the chance level (52\% correct). Surprisingly, stimuli presented only $1 \mathrm{deg}$ from fixation were judged correctly only $68 \%$ of the time. This result is in direct contrast to the results of Experiment 1, in which performance for stimuli at $1 \mathrm{deg}$ was over $90 \%$. Differences in the task probably account for the discrepancy, as the naming task involved naming 1 of a fixed set of 30 high-frequency words, whereas the lexical decision task involved discriminating between 1 of 20 high-frequency words and a visually similar nonword. The lexical decision task thus required a finer discrimination than the naming task. Response time in the no eye movement condition yielded a slope of $32 \mathrm{msec} / \mathrm{deg}$.

As in Experiment 1, performance (accuracy and response time) was superior in the eye movement condition at all of the visual angles tested. Response time for foveally presented stimuli was $118 \mathrm{msec}$ faster

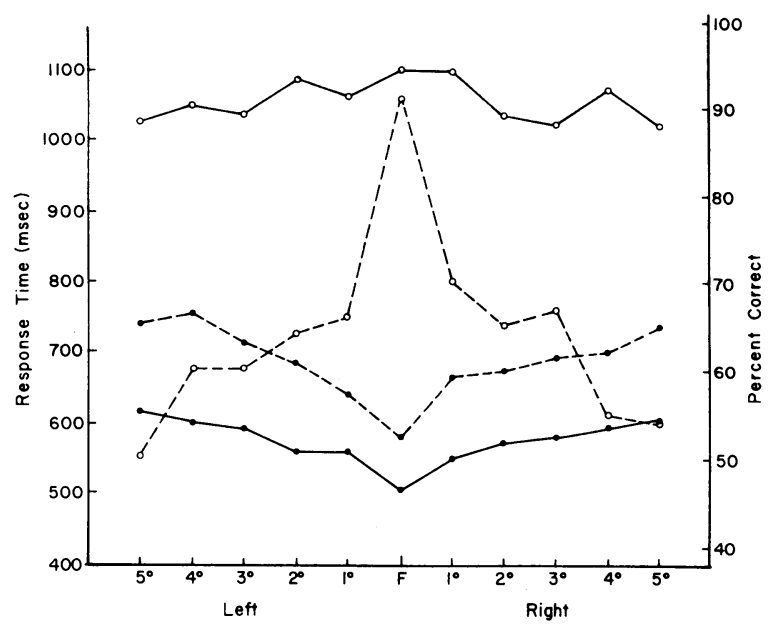

Figure 2. Response time and percent correct in the lexical decision task (Experiment 2). Open circles indicate percent correct, and filled circles indicate response time. Solid lines represent the eye movement condition, and dotted lines represent the no eye movement condition.

in the eye movement condition than in the no eye movement condition. Separate 2 by 2 by 11 analyses of variance were performed on the response time and accuracy data (again, using the arcsin transformation). Overall, response time increased as stimulus eccentricity did, yielding a main effect of eccentricity $[F(10,60)=12.25$, $\mathrm{p}<.001]$. As in Experiment 1, although response times for the no eye movement condition were longer at every eccentricity, the main effect of movement was not significant. The lack of a significant effect of movement upon response time is explained by noting that accuracy is not equivalent between the two conditions. At $5 \mathrm{deg}$, subjects performed essentially at chance in the no eye movement condition and probably were merely guessing, with latencies not much greater than in the eye movement condition. The nominal correct response times may often be good, quick guesses. A main effect of movement was evident in the accuracy data (discussed below). Unlike in Experiment 1, the Movement by Eccentricity interaction failed to reach significance. Response times for the no eye movement condition did not increase with increasing eccentricity by an amount significantly greater than the increase shown by the eye movement condition.

The accuracy data did show a large Movement by Eccentricity interaction $[\mathrm{F}(10,60)=5.62, \mathrm{p}<.001]$. Although accuracy varied little with eccentricity in the eye movement condition, it fell very sharply with increasing eccentricity in the no eye movement condition. This interaction was so large that it was reflected in the overall trends of a main effect for eccentricity reaching significance $[\mathrm{F}(10,60)=12.97, \mathrm{p}<.001]$, as well as the main effect for movement $[F(1,6)=103.53$, $\mathrm{p}<.001]$. In addition, there was a significant interaction of stimulus type (word or nonword) with eccentricity $[F(10,60)=2.23, p<.05]$, due to the fact that responses to nonwords were less accurate than were 
responses to words when presented within $2 \mathrm{deg}$ of fixation, but more accurate when presented $5 \mathrm{deg}$ from fixation. Presumably, subjects often guessed at stimuli occurring $5 \mathrm{deg}$ from fixation and were biased toward nonword responses. Furthermore, this data pattern was found mainly in the no eye movement condition (responses to nonwords in the eye movement condition were slightly less accurate at all eccentricities), thus leading to a significant Movement by Stimulus Type by Eccentricity interaction $[F(10,60)=4.32, p<.001]$.

In both of the experiments, response times to foveally presented stimuli were shorter in the eye movement condition than in the no eye movement condition. Subjects reported that suppressing eye movements while diverting attention to a parafoveal stimulus was a difficult task. This suggests that a certain amount of processing capacity may be taken up by holding the eyes in fixation. In order to verify this, we replicated Experiment 2 using blocked presentations (of the eccentricity variable). Of course, with blocked presentations, subjects knew where the stimulus would be presented, and, therefore, in the foveal condition they did not have to suppress eye movements. Under these circumstances, response times for foveally presented stimuli were slightly shorter in the nominal no eye movement condition than in the eye movement condition. Thus, it would appear that the longer response times for foveally presented stimuli in the no eye movement condition in Experiments 1 and 2 were due to the fact that holding one's eyes still in a fixation takes up processing capacity, leading to slower responses.

Foreknowledge of stimulus location also improved performance for nonfoveal stimuli. Although responses improved in speed and accuracy in the no eye movement condition, they were still inferior to responses in the eye movement condition.

\section{DISCUSSION}

The experiments reported here were undertaken to determine whether there is a tradeoff point (in terms of speed of identification) between making an eye movement and holding fixation for parafoveally presented words. Earlier, Sanders (1963) found that the functional visual field could be divided into three regions. Using a rather gross stimulus (a large dot) in a detection task, Sanders found that the target could be detected quite accurately up to about $30 \mathrm{deg}$ by the fixated eye. Targets presented at beyond approximately $30 \mathrm{deg}$ required an eye movement for accurate detection, and targets located more eccentrically than $80 \mathrm{deg}$ required an eye movement and a head movement. The data described here for a task requiring much finer discriminations indicated that subjects were always more accurate in identifying words presented parafoveally when they made an eye movement to the word. Even stimuli presented $1 \mathrm{deg}$ from fixation were identified more accurately when eye movements were made than when they were not.

In terms of speed of identification, the data indicate that parafoveal words could be identified more quickly when eye movements were made than when they were not made, even when the stimulus began (or ended, in the case of stimuli presented left of fixation) $1 \mathrm{deg}$ from fixation. Presumably, two factors account for the superiority of the eye movement performance at all visual angles tested. First, as the experiments demonstrated, holding fixation takes up a certain amount of processing capacity, increasing response time in the no eye movement condition such that subjects were approximately $100 \mathrm{msec}$ slower in responding to a foveal stimulus than they were in the eye movement condition.

Second, making an eye movement to fixate the stimulus becomes increasingly advantageous over holding fixation and using parafoveal vision as the stimulus eccentricity increases; the effectiveness of acuity declines more drastically than the time needed to redirect the eye and respond. The eye movement response time data for $1 \mathrm{deg}$ and beyond increase with a slope of $20 \mathrm{msec} / \mathrm{deg}$ in Experiment 1 and $11 \mathrm{msec} / \mathrm{deg}$ in Experiment 2. The no eye movement condition shows a slope of $85 \mathrm{msec} / \mathrm{deg}$ in Experiment 1 and $22 \mathrm{msec} / \mathrm{deg}$ in Experiment 2.

In summary, the data presented here indicate that words presented in parafoveal vision are easier to identify when eye movements are made than when they are not. This general statement is true for words presented very close to fixation, but it is especially true for words presented $2 \mathrm{deg}$ or more from fixation. Thus, in reading, it is not surprising that the average saccade length is about 2 deg of visual angle (Rayner \& McConkie, 1976).

\section{REFERENCES}

Andriessen, J. J., \& Bouma, H. Eccentric vision: Adverse interaction between line segments. Vision Research, 1976, 16, 71-78.

Bouma, H. Visual interference in the parafoveal recognition of initial and final letters of words. Vision Research, 1973, 13, 767-782.

Boum A, $\mathrm{H}$. Visual search and reading: Eye movements and functional visual field. In J. Requin (Ed.), Attention and performance VII. Hillsdale, N.J: Erlbaum, 1978.

Hilz, R., \& Cavonius, C. R. Functional organization of the peripheral retina: Sensitivity to periodic stimuli. Vision Research, 1974, 14, 1333-1337.

HinEs, M. Line spread function variation near the fovea. Vision Research, 1976, 16, 567-572.

Koenderink, J. J., Bouman, M. A., Bueno de Mesquita, A. E., \& Slappendel, S. Perimetry of contrast detection thresholds of moving spatial sine wave patterns. 1 . The near peripheral visual field (eccentricity $\left.0^{\circ}-8^{\circ}\right)$. Journal of the Optical Society of America, 1978, 68, 845-849.

Limb, J. O., \& Rubenstein, C. B. A model of threshold vision incorporating inhomogeneity of the visual field. Vision Research, 1977, 17, 571-584.

Mackworth, N. H. Visual noise causes tunnel vision. Psychonomic Science, 1965, 3, 67-68.

Millodot, M. Foveal and extra-foveal acuity with and without stabilized retinal images. British Journal of Physiological Optics, 1966, 23, 75-106.

RAYNER, K. Eye movement latencies for parafoveally presented words. Bulletin of the Psychonomic Society, 1978, 11, 13-16.

RAYNer, K., \& McConkie, G. W. What guides a reader's eye movements? Vision Research, 1976, 16, 829-837.

RAyner, K., McConkie, G. W., \& Ehrlich, S. Eye movements and integrating information across fixations. Journal of Experimental Psychology: Human Perception and Performance, 1978, 4, 529-544.

SANDERS, A. F. The selective process in the functional visual field. Soesterberg, The Netherlands: Institute for Perception, RVO-TNO, 1963.

SCHIEPERS, C. Response latency and accuracy in visual word recognition. Perception \& Psychophysics, 1980, 27, 71-81.

(Received for publication February 26, 1981.) 